\section{Genética, HLA-B27 y espondilitis anquilosante: 40 años}

\author{
PATRICIA CASTRO-SANTOS PhD ${ }^{1}$, MIGUEL A. GUTIÉRREZ ${ }^{2}$, \\ ROBERTO DÍAZ-PEÑA PhD ${ }^{1}$
}

\section{Genetics of ankylosing spondylitis}

\begin{abstract}
Ankylosing spondylitis (AS) is a prototypical inflammatory disease of the locomotor system affecting axial skeleton. It is part of the general group of spondyloarthopathies (SpA). Its strong association with histocompatibility antigen HLA-B27 is known since 1973. However, HLA-B27 contribution to AS genetic risk is approximately $16 \%$. Therefore, other genes are necessarily involved in the pathogenesis of the disease. Genomic development and the possibility of making genome wide screening have contributed enormously to the study of the disease. In this paper, we describe the actual knowledge about AS genetic risk, which has contributed to understand the influence of HLA-B27 on the etiology and pathogenesis of the disease. We also intend to foresee how these findings will result in an improvement of patients' quality of life.
\end{abstract}

(Rev Med Chile 2014; 142: 1165-1173)

Key words: Genetic predisposition to disease; HLA-B27 antigen; Spondylitis, ankylosing.

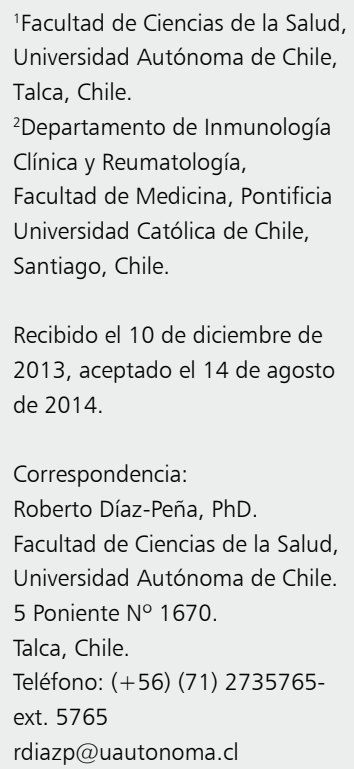

\section{Epidemiología}

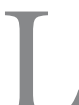

a espondilitis anquilosante (EA) es el prototipo de las enfermedades inflamatorias del aparato locomotor que afectan al esqueleto axial y que se engloban bajo el término de espondiloartropatías (SpA). La EA es una patología de etiología desconocida cuyas principales alteraciones se producen en las zonas de inserción de ligamentos y tendones en el hueso (entesis), en la membrana sinovial y en el cartílago articular. Se considera que afecta más frecuentemente a los varones (en una relación de 3:1 respecto de las mujeres) y aparece normalmente entre los 20-30 años de vida. La prevalencia de la EA varía según zona geográfica, etnia, factores genéticos y ambientales, persistiendo aún muchos factores que se desconocen ${ }^{1}$. La investigación de la EA ha dado un papel fundamental al antígeno de histocompatibilidad HLA-B27, molécula capaz de acoplar un péptido en su interior que permite la posterior activación del linfocito T, y por tanto, imprescindible para el reconocimiento de lo propio y lo ajeno por parte del sistema inmune. Las diferencias en la prevalencia de HLA-B27 explican la mayor parte de la variación en la prevalencia de la EA vista en todo el mundo ${ }^{2,3}$. Presentar en nuestro organismo este antígeno supone una probabilidad de 1-2\% de padecer EA, aunque aumenta hasta el 10-20\% si, además de ser B27-positivo, existe un familiar de primer grado que ya padece la enfermedad. Lo interesante es que alrededor del $80-95 \%$ de las personas caucasoides con EA presentan positividad para HLA B27, por lo que establecer una relación entre su presencia y el desarrollo de la enfermedad ha sido el objetivo de numerosas investigaciones. En otros grupos étnicos, la relación con el HLAB27 no es tan estrecha ${ }^{1,4,5}$. De este modo, sabemos que la presencia de HLA-B27 no significa que una persona padezca o vaya a padecer EA, pero en el caso de existir determinados síntomas, variaciones en parámetros bioquímicos y ciertos signos radiológicos, existiría un indicador que puede contribuir al diagnóstico definitivo, e incluso que podría ser útil en el diagnóstico diferencial de otras patologías. 
De esta forma y de acuerdo a los nuevos criterios de clasificación del consenso ASAS (Assessment of Spondylo arthritis International Society) para la SpA axial, la presencia de HLA-B27 permitiría clasificar como SpA a un paciente con lumbago crónico iniciado antes de los 45 años, aun en ausencia de sacroileítis (radiológica o por RNM), si presenta al menos otras 2 características de SpA. El uso de estos nuevos criterios contribuye al diagnóstico precoz de la EA, en particular de la forma de SpA no radiológica con una probabilidad post test de $86 \%{ }^{6}$.

\section{Genética de la espondilitis anquilosante}

Aunque la etiología y la patogenia de la EA sean desconocidas, existe gran cantidad de información procedente de diferentes áreas, que aporta evidencias de la participación de factores genéticos en la enfermedad. De hecho, se estima que la heredabilidad de la enfermedad supera el $90 \%{ }^{7}$. Además, esta heredabilidad es significativa cuando se tienen en cuenta distintas manifestaciones clínicas de $\mathrm{EA}^{8}$ : severidad medida por radiografías del $40 \%$, edad de comienzo de los síntomas del $62 \%$, e incluso actividad medida por BASDAI (Bath Ankylosing Spondylitis Disease Activity Index) y BASFI (Bath Ankylosing Spondylitis Functional Index), de 51 y $76 \%$, respectivamente. Todo indica que el riesgo de desarrollar EA está determinado genéticamente, y sugiere que cualquiera que sea la causa ambiental que desencadena su inicio o que influye en su desarrollo, esta es ubicua.

\section{HLA-B27}

Las moléculas HLA fueron inicialmente descubiertas por ser las responsables del rechazo de los trasplantes de tejidos, debido a la generación por parte del huésped de respuestas de linfocitos B y T dirigidas contra las moléculas HLA no propias. Sin embargo, hoy sabemos que las moléculas HLA juegan un papel esencial en la respuesta inmunológica uniendo y presentando antígenos a los linfocitos $\mathrm{T}$ en la superficie celular. La región en la que se encuentran estas moléculas HLA es muy polimórfica, contiene más de 200 loci (MHC Sequencing Consortium 1999) y alrededor del $40 \%$ de los loci que se expresan codifican moléculas relacionadas con el sistema inmune. Los diferentes loci han sido agrupados en tres regiones desde el punto de vista estructural y funcional (Figura 1). En la región de clase I se localizan los loci HLA-A, -B y -C, que son importantes pues codifican proteínas que son expresadas en la superficie de todas las células nucleadas del organismo, y su función es la de presentar antígenos a los linfocitos T citotóxicos. Sus productos poseen un alto grado de variación alélica dentro de cada locus, lo que desemboca en una gran variabilidad entre individuos. Han sido identificados 2.884 alelos HLA-A, 3.589 HLA-B y 2.375 HLA-C en diferentes poblaciones (http:// www.ebi.ac.uk/imgt/hla), siendo esta la región más polimórfica dentro del genoma humano.

Han pasado más de 40 años desde el descubrimiento de la asociación de HLA-B27 con $\mathrm{EA}^{9,10}$, y el papel patogénico del gen y su producto permanecen sin resolver. Sin embargo, este antígeno de histocompatibilidad representa el factor genético más importante de asociación con EA. Si bien durante todo este tiempo ha habido grandes avances en cuanto a la clasificación y tratamiento de la EA como de otras SpA, así como mayor conocimiento en la biología de la molécula B27, las bases moleculares de la asociación permanecen sin resolver, aunque nadie duda que esté directamente involucrada en la patogénesis de la $\mathrm{EA}^{8}$.

La asociación tiene lugar en diferentes poblaciones y grupos étnicos, en el contexto de múltiples subtipos diferentes ${ }^{4}$ (Tabla 1). Hasta el momento se han descrito más de 100 alelos diferentes de HLA-B27 (http://www.ebi.ac.uk/cgi-bin/ipd/ imgt/hla/allele.cgi) cuyo polimorfismo se localiza en unos pocos aminoácidos situados en el sitio de unión al péptido. El alelo $B^{\star 27: 05}$ ha sido encontrado mayoritariamente en todas las poblaciones estudiadas; $B^{\star} 27: 01,02,08$ y 09 han sido descritos en caucasoides, mientras que $\mathrm{B}^{\star} 27: 04,06$ y 07 solamente han sido observados en poblaciones orientales y $\mathrm{B}^{\star} 27: 03$ en poblaciones del oeste africano. No todos los subtipos HLA-B27 están igualmente asociados a enfermedad, sugiriendo que el polimorfismo podría modular la susceptibilidad a la enfermedad. Los alelos $B^{\star} 27: 02,04$ y 05 están fuertemente asociados a $\mathrm{EA}$, mientras que $B^{\star} 27: 07$ y $B^{\star} 27: 03$ se encuentran asociados con una menor prevalencia en algunas poblaciones. Existen dos alelos, $\mathrm{B}^{\star}$ 27:06 (restringido a poblaciones del sudeste asiático) y $\mathrm{B}^{\star}$ 27:09 (restringido a la población sarda), que no se han encontrado 


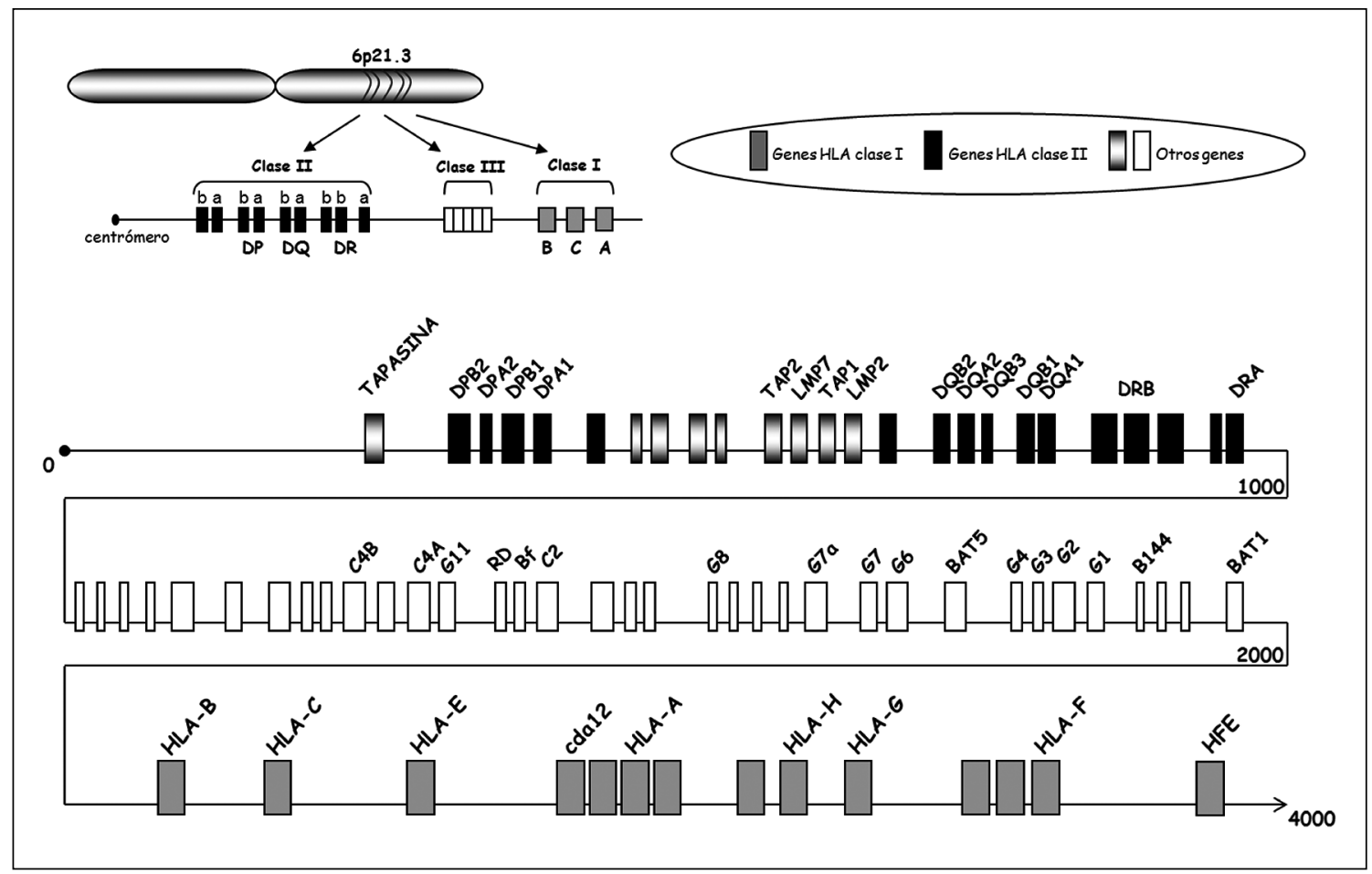

Figura 1. Mapa génico de la región HLA.

Tabla 1. Distribución poblacional y asociación a EA de los subtipos HLA-B27

\begin{tabular}{|c|c|c|}
\hline $\begin{array}{l}\text { Subtipo } \\
\text { B27 }\end{array}$ & $\begin{array}{c}\text { Asociación } \\
\text { a EA }\end{array}$ & Distribución \\
\hline$B * 27: 02$ & Sí & $\begin{array}{l}\text { Europa, Oriente medio, } \\
\text { Norte de África }\end{array}$ \\
\hline$B * 27: 03$ & Sí & Oeste de África \\
\hline$B * 27: 04$ & Sí & $\begin{array}{l}\text { China, Japón, Tailandia, } \\
\text { Indonesia y Polinesia }\end{array}$ \\
\hline$B * 27: 05$ & Sí & Mundial \\
\hline$B * 27: 06$ & No & Sureste de Asia \\
\hline$B * 27: 07$ & Sí & China, Tailandia, India \\
\hline$B * 27: 08$ & Sí & $\begin{array}{l}\text { Islas Azores, Noroeste de } \\
\text { España, Islas Británicas }\end{array}$ \\
\hline$B * 27: 09$ & No & Italia y Cerdeña \\
\hline
\end{tabular}

asociados a enfermedad ${ }^{11,12}$ (Tabla 1$)$. El resto de subtipos son muy raros, lo que hace que no se disponga de la información necesaria para llevar a cabo el estudio de su asociación.
Las proteínas HLA de clase I unen grandes repertorios peptídicos, principalmente provenientes de la degradación de proteínas endógenas, y los presentan en la superficie celular para que sean reconocidos por los linfocitos T CD8+. Así, parece lógico suponer que el papel patogénico de HLA-B27 podría estar relacionado con sus características presentadoras de antígeno y con el reconocimiento de éstos por las células T. Sin embargo, el papel que juega HLA-B27 en la EA no se ha establecido, aunque las investigaciones llevadas a cabo a lo largo de estos años han dado como resultado la existencia de hipótesis sobre cómo HLA-B27 puede llevar a EA. Existe la "hipótesis del péptido artritogénico"13, según la cual un péptido bacteriano o viral, podría ser presentado por HLA-B27 y desencadenar una respuesta mediada por linfocitos T citotóxicos (CTL).Si este péptido externo mostrase mimetismo molecular con algún péptido propio presentado por el antígeno HLAB27, algunos CTLs activados podrían eludir los mecanismos de generación de tolerancia contra ese péptido induciendo autoinmunidad, daño tisular e inflamación (Figura 2A). La idea de que en la 
secuencia de HLA-B27 se incluyan homologías con proteínas de bacterias Gram negativas ha centrado gran parte de las investigaciones en lo que respecta a la búsqueda de péptidos artritogénicos ${ }^{14}$. Se ha identificado un ligando de HLA-B27 perteneciente a su propia secuencia, que presenta similitud con una secuencia de la ADN-primasa de Chlamydia trachomatis ${ }^{15}$. Posteriormente, se expresó un fragmento proteico que incluía parte de la secuencia homóloga a B27 de esta ADN-primasa bacteriana en líneas celulares B27-positivas, probando que la construcción proteica era procesada y presentada “in vivo" por $\mathrm{B}^{\star} 27: 05^{16}$. Además, mediante inmunoproteómica comparada, se demostró que un péptido bacteriano homólogo al ligando propio de $B^{\star} 27$ era procesado y presentado endógenamente, demostrando la existencia de mimetismo molecular ${ }^{17}$. Sin embargo, a pesar de todas estas evidencias, aún no se ha podido constatar la existencia de péptidos artritogénicos.

Otras hipótesis se basan en características biológicas de HLA-B27 que no están relacionadas con la presentación antigénica convencional (Figura 2B). Se ha demostrado una tendencia de HLA-B27 a plegarse erróneamente formando homodímeros y formas no canónicas, que podrían acumularse en el retículo endoplasmático (RE) e inducirían respuestas a estrés, desencadenando procesos inflamatorios que podrían tener un papel patogénico en el desarrollo de la $\mathrm{EA}^{18}$. El plegamiento anómalo podría inducir la activación de NF- $\kappa \mathrm{B}$ y éste, a su vez, induciría producción de citoquinas proinflamatorias tales como el TNF- $\alpha$, IL-1 o IL6 , en monocitos-macrófagos ${ }^{18}$. Con el objetivo de contrarrestar el estrés celular que se produce por el acúmulo de proteínas mal plegadas o mal procesadas en el RE, en la célula se activa una ruta de señalización intracelular denominada UPR (Unfolded Protein Response) ${ }^{19}$, que desencadena la

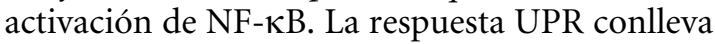
una serie de procesos encaminados a proporcionar a la célula mecanismos de supervivencia (inducción transcripcional de genes, atenuación de traducción de proteínas), entre los que se encuentra la producción de IL23 y la activación de células Th17 (vía IL-23/IL-17, comentada en el apartado "Estudios GWAS"). Existen otros estudios que han relacionado este plegamiento anómalo de HLA-B27 con la formación de homodímeros en el $\mathrm{RE}^{20,21}$, y su expresión en la superficie celular ${ }^{22}$; de forma que puedan ser reconocidos por receptores de células NK (natural killer) ${ }^{23,24}$ (Figura 2B). Entre los receptores leucocitarios que podrían reconocer las formas homodiméricas estarían LILRA1,

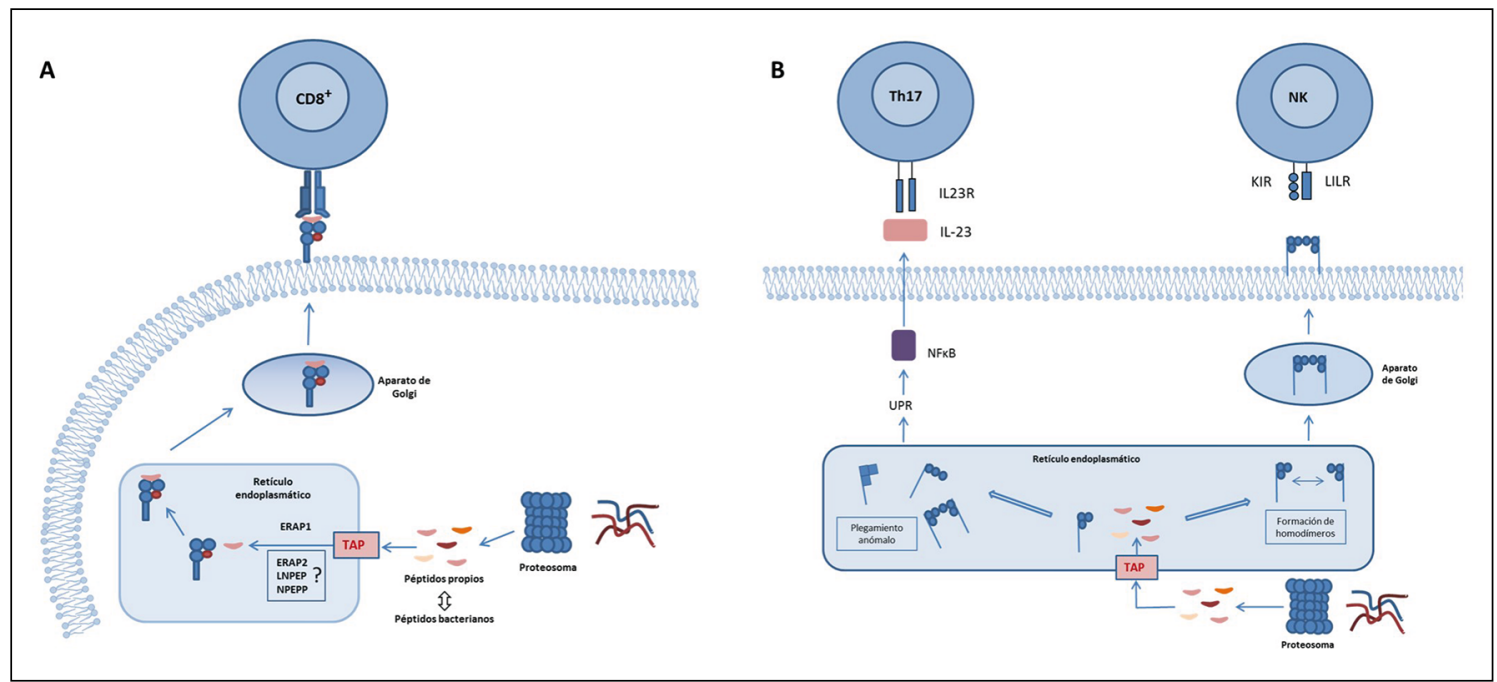

Figura 2. Hipótesis que explican la influencia de HLA-B27 en EA. A. Representación de la hipótesis del péptido artritogénico, según la cual podría existir mimetismo molecular entre péptidos propios y foráneos, que desencadenaría una respuesta celular T que podría llevar a destrucción tisular e inflamación; B. HLA-B27 tiene tendencia a plegarse erróneamente, lo que podría llevar a una respuesta proinflamatoria mediada por la ruta UPR, que derivaría en la producción de IL23 y la activación de células Th17. Otra posibilidad es que ese plegamiento anómalo lleve a la formación de homodímeros, que podrían ser reconocidos por receptores de las células NK en la superficie celular (receptores KIR y LILR). 
LILRB2, KIR3DL1 y KIR3DL2. Se ha propuesto la hipótesis mediante la cual el reconocimiento de formas canónicas (heterodímeros) y no canónicas (homodímeros) de HLA-B27 por estos receptores específicos de NK podría tener un efecto inmunomodulador en la enfermedad ${ }^{25}$.

Existen otros mecanismos por los que HLAB27 podría estar involucrado en la patogénesis de la EA. Se ha descrito que péptidos derivados de la molécula B27 podrían ser presentados por moléculas HLA de clase II (DR, DQ y DP), y ser reconocidos como autoantígenos por linfocitos CD4+ que hubiesen sido previamente activados por antígenos bacterianos ${ }^{26}$. También se han postulado otras hipótesis de asociación a EA no relacionadas con la función presentadora de antígeno de HLA-B27. Concretamente se afirma que la aparición de inflamación podría ser causada por el depósito de $\beta 2$-microglobulina $(\beta 2 \mathrm{~m})$, molécula que forma parte de la estructura de todos los receptores HLA de clase I, en las articulaciones ${ }^{27}$.

\section{Otros alelos HLA-B}

Diferentes estudios han sugerido que la presencia de otros alelos HLA-B podría influir en la susceptibilidad a $\mathrm{EA}^{28-30}$. El incremento de la frecuencia de HLA-B60 y HLA-B39, por ejemplo, se ha asociado a la susceptibilidad a desarrollar EA independientemente de B27 en pacientes de China y Japón, respectivamente ${ }^{28,29}$. Cabe destacar la asociación de HLA-B ${ }^{\star} 14: 03$, un alelo que sólo se había detectado en individuos no relacionados de Camerún mediante tipaje de rutina ${ }^{31}$, y que ha sido asociado a EA en poblaciones sub-saharianas ${ }^{30}$. Otro trabajo asoció a los alelos HLA-B ${ }^{\star} 38, \mathrm{~B}^{\star} 40 \mathrm{y}$ $\mathrm{B}^{\star} 52$ con la enfermedad ${ }^{32}$. Debido a la importancia que puede tener el reconocimiento de péptidos específicos por parte de las células T en EA, parte de los esfuerzos de las investigaciones se han destinado a buscar péptidos que puedan ser presentados por alelos HLA-B ${ }^{33}$, y en valorar plegamiento, maduración y estabilidad de alelos HLA-B ${ }^{34}$. Ambos trabajos se han centrado en $B^{\star} 27: 05$ y $B^{\star} 14: 03$. Aún no existen muchos resultados, pero no se excluye la posibilidad de que exista un mecanismo por el que, ligandos específicos de diferentes alelos HLA-B, medien en la EA. Así, serán necesarios más estudios que clarifiquen el papel de HLA-B en la susceptibilidad a padecer EA.

\section{Estudios GWAS}

Los estudios de asociación de genoma completo, o GWAS, se consideran una de las principales herramientas para identificar variantes genéticas asociadas a una enfermedad. Hasta el momento, se han realizado numerosos estudios de este tipo en diferentes patologías (http://www.genome. gov/26525384). En ellos, se genotipifican al menos 100,000 polimorfismos de una sola base (SNPs) en cohortes de más de 1.000 sujetos en la mayor parte de las ocasiones, por lo que aportan una visión objetiva de la totalidad del genoma, y una mayor probabilidad de detectar una asociación con un marcador genético. En lo que respecta a EA, se ha evidenciado que nos encontramos ante una enfermedad multifactorial con una base poligénica, en la que están involucrados genes tanto dentro como fuera del complejo mayor de histocompatibilidad, siendo HLA-B27 uno de los genes implicados.Específicamente, además de la región HLA, unos 30 loci de riesgo no-HLA han sido descritos ${ }^{35}$. En otro GWAS llevado a cabo en población china ha identificado 2 nuevos loci asociados con $\mathrm{EA}^{36}$, un polimorfismo situado en $5 q 14.3$ entre los genes EDIL3 y HAPLN1, y otro ubicado dentro del gen ANO6 en la región 12q12, aunque ninguna de estas asociaciones ha sido replicada posteriormente.

En el año 2007 se realizó el primer gran estudio de asociación genética en $\mathrm{EA}^{37}$, genotipando 14.500 SNPs no sinónimos (nsSNPs) en 1.000 pacientes EA y 1.500 controles. Entre los resultados destacables, estaba el que cinco nsSNPs de los que mostraba asociación significativa con EA estaban localizados en el gen ERAP1, que codifica una aminopeptidasa del RE. Estos resultados fueron posteriormente replicados en estudios de asociación independientes en diferentes poblacio$n^{38}{ }^{38}$, e incluso en el último GWAS llevado a cabo en $\mathrm{EA}^{35}$, mutaciones presentes en genes de otras aminopeptidasas como ERAP2, LNPEP y NPEPPS fueron asociadas con EA. ERAP1 está involucrada en el escisión de receptores de algunas citoquinas proinflamatorias, como IL1, IL6 y factor de necrosis tumoral (TNF) alfa, de la superficie celular ${ }^{39-41}$, con un papel importante en el procesamiento post-proteosomal de ligandos para moléculas MHC de clase ${ }^{42,43}$. Posteriormente, se comprobó que la asociación de ERAP1 y EA sólo tenía lugar entre individuos HLA-B27 positivos $^{44}$, sugiriendo 
un papel patogénico en la enfermedad a través de una epistasis con HLA-B27. Este hecho se ha visto respaldado por trabajos que demuestran que las variantes de ERAP1 difieren en su actividad funcional y ejercen un efecto global distinto sobre el conjunto de péptidos (peptidoma) que presenta HLA-B2 $7^{45}$. Lo que está claro es que este descubrimiento abre el camino para que futuros estudios vayan encaminados a responder a la pregunta de por qué los individuos HLA-B27-postivos tienen más riesgo de padecer EA, lo que llevará seguro a un mejor entendimiento de los mecanismos patogénicos de la enfermedad.

En el estudio de asociación llevado a cabo en el año $2007^{37}$, también se observó asociación con otro gen, IL23R, que codifica para una subunidad del receptor de la citoquina IL-23 y que se expresa en las células Th17, subclase de células T involucradas en la producción de la citoquina proinflamatoria IL-17, e implicadas en el desarrollo de enfermedades autoinmunes ${ }^{46}$. A partir de entonces se han llevado a cabo varios estudios de replicación en distintas poblaciones ${ }^{47}$, y constituyentes de la vía IL-23/IL-17 han sido asociados con $\mathrm{EA}^{48}$. En la vía de señalización JAK-STAT a través de IL23R intervienen las kinasas TYK2 y JAK2, y STAT3, $\mathrm{y}$ mutaciones en los genes que codifican estas proteínas se han encontrado asociadas con la susceptibilidad a padecer $\mathrm{EA}^{35,49}$. Lo importante es que la implicación de la vía IL-23 parece clara, y ha estimulado que existan ensayos de antagonistas de esta vía en EA. Existen evidencias de la eficacia de tratamientos con ustekinumab (anticuerpo anti-IL-12p40, una de las subunidades de IL-23) y secukimumab (anticuerpo anti-IL-17) en EA, y ensayos con tofacitinib (inhibidor JAK) y fostamatinib (inhibidor TYK2) están en marcha en la actualidad. Con el objetivo de explorar nuevas terapias para pacientes con EA, especialmente para los pacientes que no responden a inhibidores de TNF- $\alpha$, más estudios tienen que ser llevados a $\mathrm{cabo}^{50}$.

Los GWAS han supuesto una herramienta vital a la hora de localizar asociaciones no-HLA en EA (Tabla 2). Algunas de ellas involucran regiones y vías que no se creían asociadas a la enfermedad, por lo que requieren una fase de validación, e incluso alguna asociación sugerida, podría estar asociada en realidad con determinados fenotipos de la enfermedad. También habría que tener en cuenta diferentes estudios de asociación que involucran genes o regiones que no han sido encontradas en estudios GWAS, como por ejemplo los genes $\mathrm{KIR}^{51}$, y que pueden tener un papel importante en la etiopatogenia de la EA.

\section{Conclusiones}

La investigación en el campo de la genética y la genómica avanzan rápidamente. Lo ha hecho a través de la genotipificación de SNPs y los GWAS, y lo seguirá haciendo con las nuevas técnicas de secuenciación masiva que ya se utilizan en la actualidad. Una consecuencia importante de todo este conocimiento será poder considerar determinadas variaciones genéticas como biomarcadores. En algunos de estos polimorfismos se localiza el que una

Tabla 2. Principales factores genéticos asociados con EA en estudios GWAS y su función

\begin{tabular}{|lll|}
\hline Gen o región cromosómica & Asociación & Función \\
HLA-B27, ERAP1, & Confirmada & Presentación antigénica \\
RUNX3, ERAP2, LNPEP, NPEPPS & Requiere replicación & \\
IL23R, IL12B & Confirmada & Vía IL-23/Th17 \\
TYK2, IL6R, IL27 & Requiere replicación & \\
2p15, 21q22 & Confirmada & Desconocida (regiones desérticas de genes) \\
KIF21B & Confirmada & Vía NF- $\kappa \beta$ \\
PTGER4, CARD9, IL1R2 & Requiere replicación & Respuesta inmune innata \\
ANTXR2 & Requiere replicación & Posiblemente a nivel óseo \\
TNFRI/LTBR, TBKBP1 & Requiere replicación & Vía TNF \\
TBX21 & Requiere replicación & Diferenciación de T helper 1 (Th1) \\
\hline
\end{tabular}


persona tenga mayor predisposición a desarrollar una determinada enfermedad, pero lo importante no sólo será conocer el riesgo que tendremos a padecer una patología, sino que podremos llevar a cabo mecanismos de prevención con intervenciones en hábitos de vida, seguimiento específico, entre otros, con lo que introducimos el concepto de medicina personalizada y preventiva. Otro de los puntos importantes a tener en cuenta en los próximos años será la relevancia que cobrará el poseer un perfil genético en la prescripción de fármacos. El área que estudia las consecuencias que tiene la diversidad genética de una persona en la respuesta a uno u otro medicamento se denomina farmacogenética. En el campo de la Reumatología, y en concreto en la EA, el desafío en los próximos años será llevar a cabo la identificación de variantes genéticas asociadas con las distintas manifestaciones de la enfermedad. Hallazgos genéticos propios de cada población (diferentes países latinoamericanos o asiáticos), podrían explicar diferencias en la expresión clínica de la EA con respecto a otras poblaciones como la europea. Además, existen otros aspectos y áreas de investigación, como la variación en el número de copias (o CNVs), la epigenética, microRNAs, etc, que requieren de tiempo para poder establecer qué papel pueden tener en la enfermedad. Lo que está claro es que en los próximos años se obtendrán datos provenientes de las ciencias "ómicas" (genómica, proteómica, epigenómica, transcriptómica, metabolómica, ) que pueden tener impacto y relevancia clínica, y que ayuden a clarificar el papel de HLA-B27 en la EA, y la etipatogenia de la misma.

\section{Referencias}

1. Dean LE, Jones GT, MacDonald AG, Downham C, Sturrock RD, Macfarlane GJ. Global prevalence of ankylosing spondylitis. Rheumatology (Oxford) 2014; 53 (4): 650-7.

2. Gofton JP, Robinson HS, Trueman GE. Ankylosing spondylitis in a Canadian Indian population. Ann Rheum Dis 1966; 25 (6): 525-7.

3. Reveille JD, Ball EJ, Khan MA. HLA-B27 and genetic predisposing factors in spondyloarthropathies. Curr Opin Rheumatol 2001; 13 (4): 265-72.

4. González-Roces S, Álvarez MV, González S, Dieye A, Makni H, Woodfield DG, et al. HLA-B27 polymorphism and worldwide susceptibility to ankylosing spondylitis. Tissue Antigens 1997; 49 (2): 116-23.
5. Benegas M, Muñoz-Gomariz E, Font P, Burgos-Vargas $\mathrm{R}$, Chaves J, Palleiro D, et al. Comparison of the clinical expression of patients with ankylosing spondylitis from Europe and Latin America. J Rheumatol 2012; 39 (12): 2315-20.

6. Rudwaleit $M$, van der Heijde $\mathrm{D}$, Landewé $\mathrm{R}$, Listing J, Akkoc N, Brandt J, et al. The development of Assessment of SpondyloArthritis international Society classification criteria for axial spondyloarthritis (part II): validation and final selection. Ann Rheum Dis 2009; 68 (6): 777-83.

7. Brown MA, Kennedy LG, MacGregor AJ, Darke C, Duncan E, Shatford JL, et al. Susceptibility to ankylosing spondylitis in twins: the role of genes, HLA, and the environment. Arthritis Rheum 1997; 40: 1823-8.

8. Thomas GP, Brown MA. Genetics and genomics of ankylosing spondylitis. Immunol Rev 2010; 10 (52): 263-71.

9. Brewerton DA, Hart FD, Nicholls A, Caffrey M, James DC, Sturrock RD. Ankylosing spondylitis and HL-A 27. Lancet 1973; 1: 904-7.

10. Schlosstein L, Terasaki PI, Bluestone R, Pearson CM. High association of an HL-A antigen, W27, with ankylosing spondylitis. N Engl J Med 1973; 288: 704-6.

11. López-Larrea C, Sujirachato K, Mehra NK, Chiewsilp $\mathrm{P}$, Isarangkura D, Kanga U, et al. HLA-B27 subtypes in Asian patients with ankylosing spondylitis. Evidence for new associations. Tissue Antigens 1995; 45: 169-76.

12. Paladini F, Taccari E, Fiorillo MT, Cauli A, Passiu G, Mathieu A, et al. Distribution of HLA-B27 subtypes in Sardinia and continental Italy and their association with spondylarthropathies. Arthritis Rheum 2005; 52 (10): 3319-21.

13. Benjamin R, Parham P. Guilt by association: HLA-B27 and ankylosing spondylitis. Immunol Today 1990; 11 (4): 137-42.

14. Scofield RH, Warren WL, Koelsch G, Harley JB. A hypothesis for the HLA-B27 immune dysregulation in spondyloarthropathy: contributions from enteric organisms, B27 structure, peptides bound by B27, and convergent evolution. Proc Natl Acad Sci USA 1993; 90 (20): 9330-4.

15. Ramos M, Álvarez I, Sesma L, Logean A, Rognan D, López de Castro JA. Molecular mimicry of an HLA-B27derived ligand of arthritis-linked subtypes with chlamydial proteins. J Biol Chem 2002; 277 (40): 37573-81.

16. Cragnolini JJ, de Castro JA. Identification of endogenously presented peptides from Chlamydia trachomatis with high homology to human proteins and to a natural self-ligand of HLA-B27. Mol Cell Proteomics 2008; 7 (1): $170-80$. 
17. Cragnolini JJ, García-Medel N, de Castro JA. Endogenous processing and presentation of T-cell epitopes from Chlamydia trachomatis with relevance in HLA-B27-associated reactive arthritis. Mol Cell Proteomics 2009; 8 (8): 1850-9.

18. Colbert RA, DeLay ML, Layh-Schmitt G, Sowders DP. HLA-B27 misfolding and spondyloarthropathies. Adv Exp Med Biol 2009; 649: 217-34.

19. Colbert RA, DeLay ML, Layh-Schmitt G, Sowders DP. HLA-B27 misfolding and spondyloarthropathies. Adv Exp Med Biol 2009; 649: 217-34.

20. Dangoria NS, DeLay ML, Kingsbury DJ, Mear JP, Uchanska-Ziegler B, Ziegler A, et al. HLA-B27 misfolding is associated with aberrant intermolecular disulfide bond formation (dimerization) in the endoplasmic reticulum. J Biol Chem 2002; 277: 23459-68.

21. Antoniou AN, Ford S, Taurog JD, Butcher GW, Powis SJ. Formation of HLA-B27 homodimers and their relationship to assembly kinetics. J Biol Chem 2004; 279: 8895-902.

22. Bird LA, PehCA, Kollnberger S, Elliott T, McMichael AJ, Bowness P. Lymphoblastoid cells express HLA-B27 homodimers both intracellularly and at the cell surface following endosomal recycling. Eur J Immunol 2003; 33: 748-59.

23. Allen RL, Raine T, Haude A, Trowsdale J, Wilson MJ. Leukocyte receptor complex-encoded immunomodulatory receptors show differing specificity for alternative HLA-B27 structures. J Immunol 2001; 167: 5543-7.

24. Kollnberger S, Bird L, Sun MY, Retiere C, Braud VM, McMichael A, et al. Cell-surface expression and immune receptor recognition of HLA-B27 homodimers. Arthritis Rheum 2002; 46: 2972-82.

25. Allen RL, Trowsdale J. Recognition of classical and heavy chain forms of HLA-B27 by leukocyte receptors. Curr Mol Med 2004; 4: 59-65.

26. Boyle LH, Goodall JC, Opat SS, Gaston JS. The recognition of HLA-B27 by human CD4(+) T lymphocytes. J Immunol 2001; 167 (5): 2619-24.

27. Uchanska-Ziegler B, Ziegler A. Ankylosing spondylitis: a beta2m-deposition disease? Trends Immunol 2003; 24 (2): 73-6.

28. Wei JC, Tsai WC, Lin HS, Tsai CY, Chou CT. HLA-B60 and $\mathrm{B} 61$ are strongly associated with ankylosing spondylitis in HLA-B27-negative Taiwan Chinese patients. Rheumatology (Oxford) 2004; 43 (7): 839-42.

29. Yamaguchi A, Tsuchiya N, Mitsui H, Shiota M, Ogawa A, Tokunaga $\mathrm{K}$, et al. Association of HLA-B39 with HLA-B27-negative ankylosing spondylitis and pauciarticular juvenile rheumatoid arthritis in Japanese patients. Evidence for a role of the peptide-anchoring B pocket.
Arthritis Rheum 1995; 38 (11): 1672-7.

30. Díaz-Peña R, Ouédraogo DD, López-Vázquez A, Sawadogo SA, López-Larrea C. Ankylosing spondylitis in three Sub-Saharan populations: HLA-B ${ }^{\star} 27$ and HLA-B $^{\star} 14$ contribution. Tissue Antigens $2012 ; 80$ (1): 14-5.

31. Ellis JM, Mack SJ, Leke RF, Quakyi I, Johnson AH, Hurley CK. Diversity is demonstrated in class I HLA-A and HLA-B alleles in Cameroon, Africa: description of HLA-A ${ }^{\star} 03012,{ }^{\star} 2612,{ }^{\star} 3006$ and HLA-B ${ }^{\star} 1403,{ }^{\star} 4016$, ${ }^{*} 4703$. Tissue Antigens 2000; 56 (4): 291-302.

32. Brown MA, Jin R, Wordsworth BP, Stone M, Millicent W, Wards MM, et al. HLA Class I and II associations of ankylosing spondylitis. Arthritis Rheum 2009; 60 (Suppl 10): 1171.

33. Merino E, Montserrat V, Paradela A, López de Castro JA. Two HLA-B14 subtypes $\left(B^{\star} 1402\right.$ and $\left.B^{\star} 1403\right)$ differentially associated with ankylosing spondylitis differ substantially in peptide specificity but have limited peptide and T-cell epitope sharing with HLA-B27. J Biol Chem 2005; 280 (43): 35868-80.

34. Merino E, Galocha B, Vázquez MN, López de Castro JA. Disparate folding and stability of the ankylosing spondylitis-associated HLA-B ${ }^{\star} 1403$ and $\mathrm{B}^{\star} 2705$ proteins. Arthritis Rheum 2008; 58 (12): 3693-704.

35. International Genetics of Ankylosing Spondylitis Consortium (IGAS), Cortes A, Hadler J, Pointon JP, Robinson PC, Karaderi T, et al. Identification of multiple risk variants for ankylosing spondylitis through high-density genotyping of immune-related loci. Nat Genet 2013; 45 (7): $730-8$.

36. Lin Z, Bei JX, Shen M, Li Q, Liao Z, Zhang Y, et al. A genome-wide association study in Han Chinese identifies new susceptibility loci for ankylosing spondylitis. Nat Genet 2011; 44 (1): 73-7.

37. Wellcome Trust Case Control Consortium (WTCCC); Australo-Anglo-American Spondylitis Consortium (TASC), Burton PR, Clayton DG, Cardon LR, Craddock $\mathrm{N}$, et al. Association scan of 14,500 nonsynonymous SNPs in four diseases identifies autoimmunity variants. Nat Genet 2007; 39 (11): 1329-37.

38. Keidel S, Chen L, Pointon J, Wordsworth P. ERAP1 and ankylosing spondylitis. Curr Opin Immunol 2013; 25 (1): 97-102.

39. Cui X, Hawari F, Alsaaty S, Lawrence M, Combs CA, Geng W, et al. Identification of ARTS-1 as a novel TNFR1-binding protein that promotes TNFR1 ectodomain shedding. J Clin Invest 2002; 110 (4): 515-26.

40. Cui X, Rouhani FN, Hawari F, Levine SJ. An aminopeptidase, ARTS-1, is required for interleukin-6 receptor shedding. J Biol Chem 2003; 278 (31): 28677-85. 
41. Cui X, Rouhani FN, Hawari F, Levine SJ. Shedding of the type II IL-1 decoy receptor requires a multifunctional aminopeptidase, aminopeptidase regulator of TNF receptor type 1 shedding. J Immunol 2003; 171 (12): 6814-9.

42. Saric T, Chang SC, Hattori A, York IA, Markant S, Rock $\mathrm{KL}$, et al. An IFN-gamma-induced aminopeptidase in the ER, ERAP1, trims precursors to MHC class Ipresented peptides. Nat Immunol 2002; 3 (12): 1169-76.

43. Serwold T, Gonzalez F, Kim J, Jacob R, Shastri N. ERAAP customizes peptides for MHC class I molecules in the endoplasmic reticulum. Nature 2002; 419 (6906): 480-3.

44. Evans DM, Spencer CC, Pointon JJ, Su Z, Harvey D, Kochan G, et al. Interaction between ERAP1 and HLA-B27 in ankylosing spondylitis implicates peptide handling in the mechanism for HLA-B27 in disease susceptibility. Nat Genet 2011; 43 (8): 761-7.

45. García-Medel N, Sanz-Bravo A, Van Nguyen D, Galocha B, Gómez-Molina P, Martín-Esteban A, et al. Functional interaction of the ankylosing spondylitis-associated endoplasmic reticulum aminopeptidase 1 polymorphism and HLA-B27 in vivo. Mol Cell Proteomics 2012; 11 (11): 1416-29.
46. Park $\mathrm{H}$, Li Z, Yang XO, Chang SH, Nurieva R, Wang $\mathrm{YH}$, et al. A distinct lineage of CD4 $\mathrm{T}$ cells regulates tissue inflammation by producing interleukin 17. Nat Immunol 2005; 6 (11): 1133-41.

47. Haroon N. Endoplasmic reticulum aminopeptidase 1 and interleukin-23 receptor in ankylosing spondylitis. Curr Rheumatol Rep 2012; 14 (5): 383-9.

48. Di Meglio P, Di Cesare A, Laggner U, Chu CC, Napolitano L, Villanova F, et al. The IL23R R381Q gene variant protects against immune-mediated diseases by impairing IL-23-induced Th17 effector response in humans. PLoS One 2011; 6 (2): e17160.

49. Danoy P, Pryce K, Hadler J, Bradbury LA, Farrar C, Pointon J, et al. Association of variants at $1 \mathrm{q} 32$ and STAT3 with ankylosing spondylitis suggests genetic overlap with Crohn's disease. PLoS Genet 2010; 6 (12): e1001195.

50. Tsui FW, Tsui HW, Akram A, Haroon N, Inman RD. The genetic basis of ankylosing spondylitis: new insights into disease pathogenesis. Appl Clin Genet 2014; 7: 10515.

51. Díaz-Peña R, Blanco-Gelaz MA, López-Larrea C. KIR genes and their role in spondyloarthropathies. Adv Exp Med Biol 2009; 649: 286-99. 